Check for updates

The BMJ

Cite this as: BMJ 2021;373:n1203 http://dx.doi.org/10.1136/bmi.n1203 Published: 11 May 2021

\section{Covid-19: Caution urged while lockdown eases in England because of vaccine success}

\section{Zosia Kmietowicz}

The effectiveness of covid vaccines in reducing infections, hospital admissions, and deaths means that lockdown restrictions can be eased "very considerably" from next week, the prime minister has announced, although he urged people to "exercise caution and common sense."

Pubs and restaurants will be able to serve customers indoors from 17 May, theatres, cinemas, and other indoor venues can open, and up to six people or two households can socialise inside.

Boris Johnson said on 10 May that the changes were possible because four tests for easing restrictions had been met, namely: successful vaccine rollout, evidence of vaccine effectiveness, control of infection rates, and no changes in new variants.

On the same day no deaths from covid-19 were recorded in England, Scotland, or Northern Ireland, although four were reported in Wales. Altogether 2357 new cases were reported in the UK, while the number of people in hospital stood at around 1300, the level seen at the end of July and down from a high of 38 ooo in January.

Real world data released by Public Health England also showed that a single dose of either the Pfizer-BioNTech or Oxford-AstraZeneca vaccine reduced the risk of dying from covid-19 by $80 \%$ when compared with no vaccination. ${ }^{1}$

Researchers had linked covid-19 testing and mortality data between December and April to calculate the effects of vaccines among 48 ooo people aged 70 or over, $20 \%$ of whom were vaccinated. They reported their results as preprints that have not been peer reviewed. ${ }^{12}$

They found that one dose of the Pfizer or AstraZeneca vaccine had similar levels of protection against mortality, at $44 \%$ and $55 \%$, respectively (hazard ratios, in a comparison with unvaccinated cases, of 0.56 (95\% confidence interval 0.47 to 0.68 ) and 0.45 (0.34 to 0.59)). When combined with the protection the vaccines offer against becoming infected with SARS-CoV-2 in the first place, the protection against death rose to around $80 \%$.

Among people who had two doses of the Pfizer vaccine the protection against mortality was around $69 \%$ (hazard ratio 0.31 (0.14 to 0.69)), which, when combined with the reduced risk of becoming infected, was equivalent to an estimated protection against mortality of $97 \%$. Corresponding data on two doses of the AstraZeneca vaccine are not yet available.

A separate analysis found that one dose of the AstraZeneca or Pfizer vaccine reduced hospital admissions of people over 80 by $73 \%$ and $81 \%$, respectively, while two doses of the Pfizer-BioNTech vaccine was $93 \%$ effective. ${ }^{2}$

The lower threat of covid-19 led the chief medical officers of the UK to reduce the covid alert level from 4 to 3 , meaning that transmission of the virus is no longer deemed to be high or rising exponentially.

However, pockets of high transmission remain. Surge testing is taking place in Kensington, London, because of a "small number" of cases of the South African variant, B.1.351, and in Bolton after a cluster of cases of the Indian variant, B.1.617 was discovered.

At the Downing Street press conference Chris Whitty, England's chief medical officer, said that the number of UK cases of the variant first identified in India had risen sharply in recent weeks and could be more transmissible than the Kent variant, B.1.1.7, that is now dominant in the UK.

In documents released on 10 May the Scientific Advisory Group on Emergencies pointed out that lifting restrictions on people mixing made controlling transmission of the virus more difficult. 3 "Depending on its characteristics, a variant either with increased transmissibility or which substantially evades immunity could easily cause a significant wave of hospitalisations or deaths of a similar or larger magnitude than that seen in January 2021.”

Commenting on vaccination effectiveness, Jonathan Ball, professor of molecular virology at the University of Nottingham, said that the new data showed an "incredible outcome, as it paves a way out of this, and future, lockdowns."

He added, "To realise the full benefits, we need to get as many people vaccinated as possible. Then, when this has been achieved we should move towards good surveillance and monitoring vaccine effectiveness instead of relying on isolation and other restrictive measures, as we learn to live with the virus."

Bernal JL, Andrews N, Gower C, et al. Effectiveness of BNT162b2 mRNA vaccine and ChAdOx1 adenovirus vector vaccine on mortality following covid-19. https://khub.net/documents/135939561/430986542/Effectiveness+of+BNT162b2+mRNA+vaccine+and+ChAdOx1+adenovirus+vector+vaccine+on+mortality+following+COVID-19.pdf/9884d371-8cc8-913c-211cc2d7ce4dd1c3

Ismail SA, Vilaplana TG, Elgohari S, et al. Effectiveness of BNT162b2 mRNA and ChAdOX1 adenovirus vector COVID-19 vaccines on risk of hospitalisation among older adults in England: an observational study using surveillance data. https://khub.net/documents/135939561/430986542/Effectiveness+of+BNT162b2+mRNA+and+ChAdOx1+adenovirus+vector+COVID19+vaccines+on+risk+of+hospitalisation+among+older+adults+in+England.pdf/9e18c525-dde6-5ee4-1537-91427798686b.

3 SPI-M-O. Summary of further modelling of easing restrictions-roadmap step 3, 5 May 2021. https://assets.publishing.service.gov.uk/government/up loads/system/uploads/attachment_data/file/984521/S1227_SPI-MO_Summary_of_further_modelling_of_easing_restrictions_Roadmap_Step_3_2_.pdf. 
This article is made freely available for use in accordance with BMI's website terms and conditions for the duration of the covid-19 pandemic or until otherwise determined by BMJ. You may use, download and print the article for any lawful, non-commercial purpose (including text and data mining) provided

that all copyright notices and trade marks are retained. 